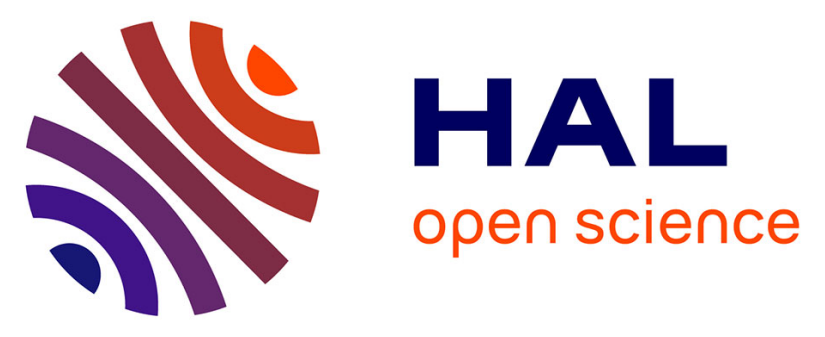

\title{
Pigtailed Electro-Optic Sensor for Time and Space Resolved Dielectric Barrier Discharges Analysis
}

Farah Aljammal, Gwenaël Gaborit, Maxime Bernier, Sylvain Iséni, Laurence Galtier, Guillaume Revillod, Lionel Duvillaret

\section{To cite this version:}

Farah Aljammal, Gwenaël Gaborit, Maxime Bernier, Sylvain Iséni, Laurence Galtier, et al.. Pigtailed Electro-Optic Sensor for Time and Space Resolved Dielectric Barrier Discharges Analysis. IEEE Transactions on Instrumentation and Measurement, 2021, 70, pp.1-9. 10.1109/TIM.2021.3107037 . hal-03326481

\section{HAL Id: hal-03326481 \\ https://hal.science/hal-03326481}

Submitted on 1 Oct 2021

HAL is a multi-disciplinary open access archive for the deposit and dissemination of scientific research documents, whether they are published or not. The documents may come from teaching and research institutions in France or abroad, or from public or private research centers.
L'archive ouverte pluridisciplinaire HAL, est destinée au dépôt et à la diffusion de documents scientifiques de niveau recherche, publiés ou non, émanant des établissements d'enseignement et de recherche français ou étrangers, des laboratoires publics ou privés. 


\title{
Pigtailed Electrooptic Sensor for Time- and Space-Resolved Dielectric Barrier Discharges Analysis
}

\author{
Farah Aljammal, Gwenaël Gaborit, Maxime Bernier, Sylvain Iséni, Laurence Galtier,
} Guillaume Revillod, and Lionel Duvillaret

\begin{abstract}
We here demonstrate the potentialities of pigtailed electrooptic probes to perform vectorial electric field characterizations of dielectric barrier discharges (DBDs). The analysis leads to the accurate determination of the breakdown voltage and the associated electric field in the vicinity of the discharge. A polarimetric analysis of the DBDs is proposed due to real-time measurement of longitudinal and radial components of the electric field vector. Moreover, the transition from capacitive to resistive behavior is characterized at the breakdown field.
\end{abstract}

Index Terms-Dielectric barrier discharges (DBDs), electric field measurement, electrical discharges, electrooptic (EO) measurement, optical sensor.

\section{INTRODUCTION}

D IELECTRIC barrier discharges (DBDs) are nonequilibrium low-temperature discharges and constitute simple devices to generate cold atmospheric plasmas [1] and partially ionized gases [2], [3]. DBDs operate in a wide range of pressures [4], [5] in various gases [6]-[9]. DBDs, also known as silent discharges, are characterized by the presence of at least one dielectric barrier in the discharge gap separating the electrodes [10]. Currently, the applications purposes concerned by ozone formation [11]-[14], treatment of exhaust gases [15], [16], ultraviolet or vacuum ultraviolet radiation generation [17]-[19], surface modification [20], [21], plasma jet production [22]-[24], and sterilization and biomedical applications [25], [26] make DBDs processing attractive for plenty of industrial applications. The plasma-chemical synthesis of ozone from either pure oxygen or air has become one of the major industrial applications of DBDs [27], [28]. In the same manner, DBDs can also modify the chemical

Manuscript received January 22, 2021; revised June 17, 2021; accepted July 28, 2021. Date of publication August 24, 2021; date of current version September 14, 2021. The Associate Editor coordinating the review process was Yuya Koyama. (Corresponding author: Farah Aljammal.)

Farah Aljammal and Maxime Bernier are with the IMEP-LAHC laboratory, Université de Grenoble Alpes, 38400 Saint-Martin-d'Héres, France, and also with the Université Savoie-Mont-Blanc, 73376 Le Bourget-du-Lac cedex, France (e-mail: farah.al-jammal@univ-smb.fr).

Gwenaël Gaborit is with the IMEP-LAHC laboratory, Université SavoieMont-Blanc, 73376 Le Bourget-du-Lac cedex, France, and also with Kapteos, 73800 Sainte-Hélène-du-Lac, France (e-mail: gwenael.gaborit@kapteos.com).

Sylvain Iséni is with GREMI, UMR7344 CNRS, Université d'Orléans, 45067 Orléans Cedex 2, France.

Laurence Galtier, Guillaume Revillod, and Lionel Duvillaret are with Kapteos, 73800 Sainte-Hélène-du-Lac, France.

Digital Object Identifier 10.1109/TIM.2021.3107037 composition of liquids for treatment applications, such as tap water depollution and more generally for plasma activated water treatment [29]. Indeed, plasma-liquid interactions have recently drawn considerable attention in the field of water treatment: numerous studies have indeed shown the effectiveness of plasma DBD activation to decontaminate water, e.g., removing pesticides [30], phenols [31], or pharmaceutical compounds [32], [33].

Electric fields ( $E$-field) have been a great topic of interest and one of the critical parameters to be analyzed for a wide variety of applications: target localization [34], nondestructive inspection [35], electromagnetic compatibility [36], and bioelectromagnetism [37]. Currently, antennas constitute the reference for $E$-field analysis [34], [35] due to their good sensitivity and dynamics. However, antennas are limited in their operating range, mainly due to their dimensions and resonant conductive parts (high induced perturbations and low bandwidths) [38], [39]. Contrarily, fully dielectric electrooptic (EO) sensors are endowed with huge bandwidths, small induced perturbation, and high spatial resolution to the detriment of sensitivity [40]. Fully dielectric and millimeter-sized EO probes, based on the Pockels effect, also called linear EO effect, have been reported in [41]. They present a frequency bandwidth spreading from $30 \mathrm{~Hz}$ up to several $\mathrm{GHz}$, a dynamic range higher than $130 \mathrm{~dB}$, a sensitivity much better than $1 \mathrm{~V} / \mathrm{m}$, a $1-\mathrm{dB}$ compression point exceeding several $100 \mathrm{kV} / \mathrm{m}$, and a spatial resolution lower than $1 \mathrm{~mm}^{3}$. Those pigtailed EO sensors constitute handful tools suitable for many applications, especially for accurate and vectorial measurements of high $E$-fields in harsh environments.

The electric field is investigated in the close vicinity of a filamentary microplasma produced with a DBD configuration. In this article, we demonstrate the ability of these EO transducers to provide a complete vectorial analysis of the $E$-field induced by the high voltage source that initiates the ionization process, on the one hand, and by the plasma itself, on the other hand. In this work, we focus on the influence of the water electrode on the characteristic of the electric field time and space dependent. The interaction of plasma and liquid (particularly aqueous solution) is of high interest for biomedical and biological applications where water molecules are playing a key role in the production of reactive oxygen species as well as ions and water clusters [29], [42], [43]. The 


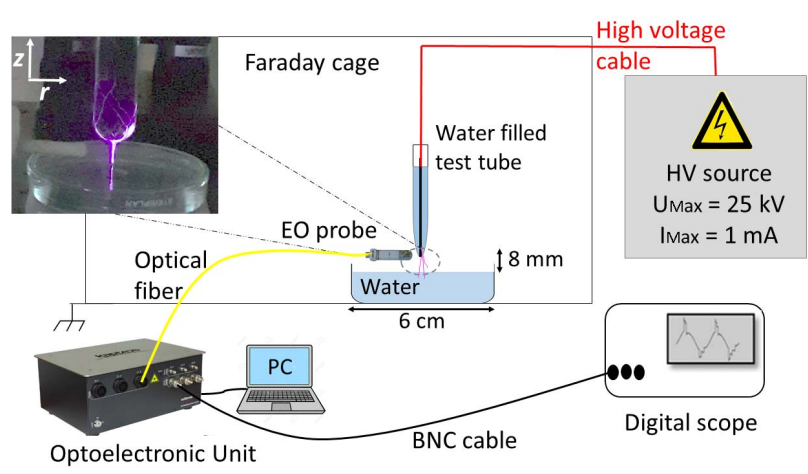

Fig. 1. Schematic of the experimental setup constituted of an ac high voltage source, an EO probe, and an optoelectronic unit from Kapteos company, a digital oscilloscope, and a DBDs reactor made of electrodes immersed in water.

EO probe is used in this context of plasma-liquid interaction in order to get better insight on the $E$-field dynamics in time and space. The results will support nonequilibrium kinetic modeling of atmospheric pressure plasmas involving liquids since the electron energy distribution function is directly correlated with the $E$-field in space and time [44]. We are interested in studying and analyzing the induced electric field of the DBDs, regarding their importance and their wide variety of applications. Considering their performances, EO sensors fulfill the requirements for the plasma analysis, such as Stark polarization spectroscopy or laser-based techniques [47], [48]. This article is divided into two sections: the first section is dedicated to the measurement configuration and the experimental set-up, while the second shows how real-time and vectorial analysis of the $E$-field associated with the DBDs can be performed by an EO probe.

\section{EXPERIMENTAl Setup}

The vectorial measurement of the electric field is here based on the first order electro-optical effect also called the Pockels effect. More precisely, the field strength and field direction induce modifications on the eigenrefractive indices of a noncentrosymmetric crystal [45]. These modifications are read by a laser probe beam, which sees its polarization state modulated. This polarization state modulation carries the information of one field vector component and is converted into a modulation of optical power and then into a modulated photocurrent due to a polarizer and a photodiode, respectively [46]. The experimental bench is presented in Fig. 1.

An ac voltage is imposed by a high voltage source to a metallic needle immersed in a test tube (diameter $1.2 \mathrm{~cm}$ and length $14 \mathrm{~cm}$ ) filled with water, while a Petri dish (diameter $6 \mathrm{~cm}$ ) filled with tap water is superimposed to the grounded electrode. The voltage function generator delivers a sector-synchronized ac voltage $(50 \mathrm{~Hz})$ whose rms magnitude is adjustable between 1 and $25 \mathrm{kVrms}$. These electrodes surrounded by dielectric barriers separated each other by $8 \mathrm{~mm}$ constitute the DBD reactor. The vectorial $E$-field generated by the above described DBD reactor is then measured by using either one EO probe or by a pair of crossed EO probes. Note that since EO probes are polarization-sensitive along one

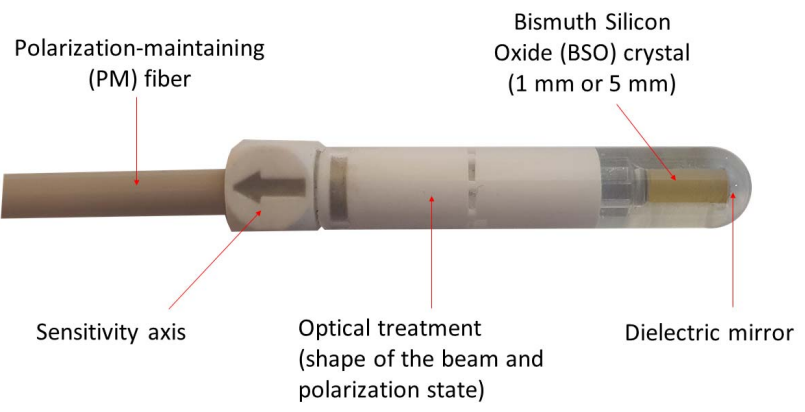

Fig. 2. Photograph showing the Kapteos pigtailed probe involving the transducer itself which is an EO crystal (bismuth silicone oxide also called BSO). Sensitivity axis of the longitudinal probe, which corresponds to the field vector component, is given by the arrow along its symmetry axis.

perfectly known axis, this latter configuration (two crossed EO probes) permits a polarimetric measurement of the $E$-field as described in Section III. Each EO probe (see Fig. 2) consists of a pigtailed optical arrangement [40], [49] allowing a noninvasive and accurate remote measurement up to more than $10 \mathrm{~m}$ in almost any environment (air, liquids, and gases) under harsh conditions and independently of temperature. The probe shown in Fig. 2 is a longitudinal probe dedicated to the measurement of the field vector component along its symmetry axis. The packaging of the sensor is here dedicated for field analysis is low permittivity media. Within the probe, a gradient index lens and a quarter-wave plate manage shape of the beam and the optical polarization state, respectively [38].

In the present case, the probes are separated by a 5-m-long polarization-maintaining (PM) fiber from the Kapteos eoSense converter. It involves first the distributed feedback (DFB) laser feeding the probe. This laser provides a low noise CW optical wave at a vacuum wavelength of $1.5 \mu \mathrm{m}$. For the detection, a photodiode performs the linear conversion between the optical modulation induced by the field and the useful electrical signal recordable on the digital oscilloscope. The bandwidth of the detection is here limited to $30 \mathrm{MHz}$ in order to avoid the integration of the noise over a wider bandwidth. This optoelectronic unit delivers finally an analog voltage proportional to the $E$-field component over a bandwidth spreading from $30 \mathrm{~Hz}$ to $30 \mathrm{MHz}$, with the upper frequency cutoff only limited by the photodiode. The optoelectronic converter measures also in real time the mean optical power in order to compensate for any parasitic losses (eventually induced by temperature drift, curvature radius of the optical fibers, mechanical stress on the optical arrangement, and so on). The signal is recorded using a digital oscilloscope with a sampling rate of $1 \mathrm{GHz}$. The whole EO system (probe coupled to optoelectronic unit) is fully characterized. Results concerning linearity and frequency bandwidth are given in Fig. 3.

The response of the longitudinal probe is given in Fig. 3(a) This curve allows to extract the minimum detectable field, which is here much lower than $1 \mathrm{Vrms} / \mathrm{m}$. Moreover, this calibration curve allows to extract the antenna factor $\mathrm{AF}$, which gives the link between a recorded voltage and the actual field strength. Here, the antenna factor is $A F=97.1 \mathrm{~dB} / \mathrm{m}$. The standard deviation of the measurement is weaker than 
a)

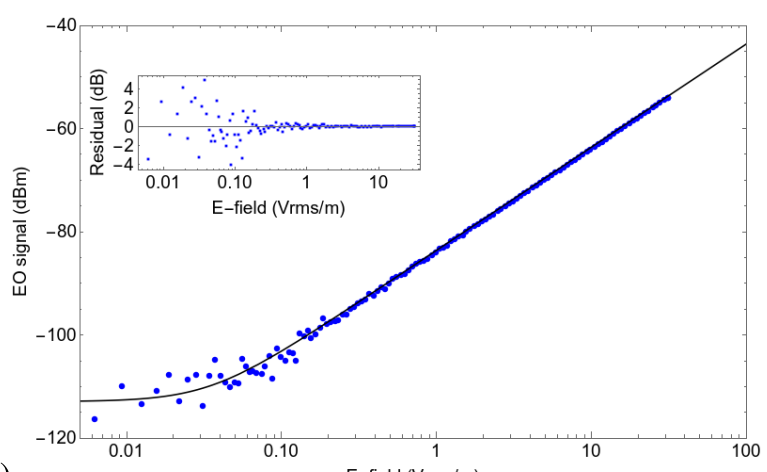

b)

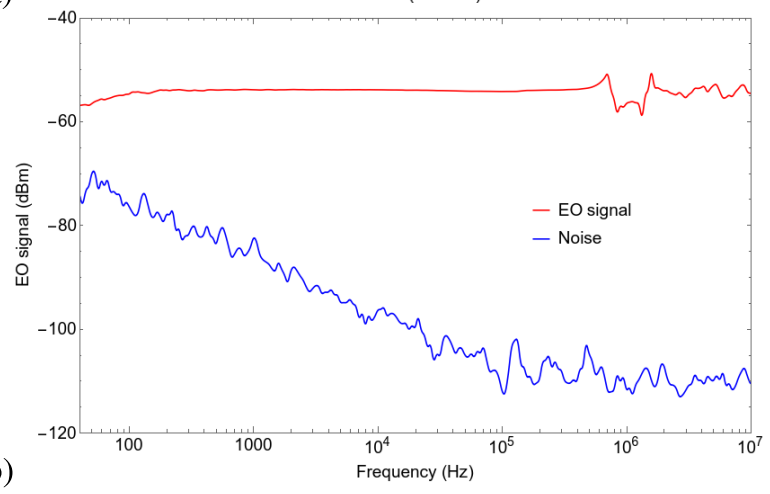

Fig. 3. (a) Typical response with respect to the field strength. Blue dots correspond to the measured data and black line corresponds to the fitting curve. The residue between the measurement and the fitting curve is given in inset of the figure. (b) Frequency response of the system.

$0.2 \mathrm{~dB}$ for field strength higher than $10 \mathrm{~V} / \mathrm{m}$. Moreover, the EO sensor exhibits a dynamic range exceeding $120 \mathrm{~dB}$ up several MV/m [38], [39]. The frequency response is flat up several $100 \mathrm{kHz}$ and then exhibits some resonances induced by piezoelectric effects. This small lack of flattness does not affect our results due to the considered bandwidth for the DBD analysis.

\section{EXPerimental Results: Transient MEASUREMENT AND ANALYSiS OF THE FIELD SURROUNDING THE DBD}

\section{A. Voltage Threshold and Simulation Analysis of the DBD Configuration}

The front of the ionization waves generated in the DBD reactor relies intrinsically on the creation of an intense $E$-field peak from which one can precisely determine the voltage threshold above which discharges occur in the reactor. In this measurement campaign, the transient evolution of the radial component $E_{r}$ is measured with an EO probe placed between the electrodes (needle and dish). Fig. 4 represents two recorded signals when the DBD reactor is powered with 16 and $18 \mathrm{kVrms}$ (just below and above the threshold voltage).

Before threshold, the radial field is a sinusoidal signal such that voltage and field are proportional to each other with a maximum field strength of the order of a few $100 \mathrm{kV} / \mathrm{m}$. Above threshold, a dramatic increase of the field is observed and the temporal profile becomes no more linear with the applied voltage. The field reaches a value of the order of $1 \mathrm{MV} / \mathrm{m}$.

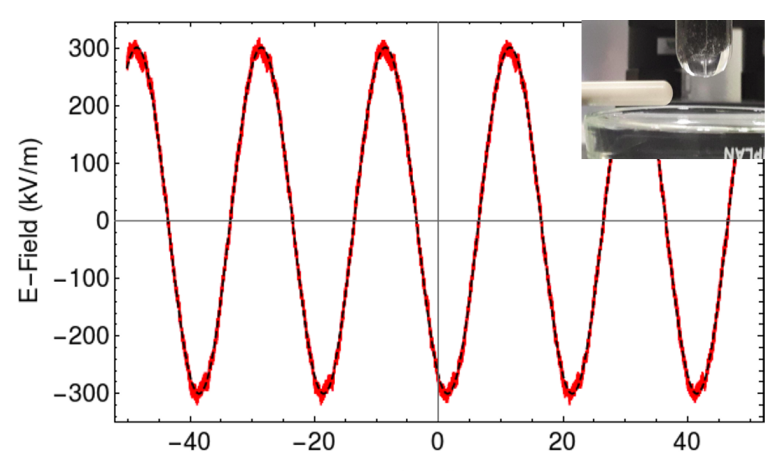

a)

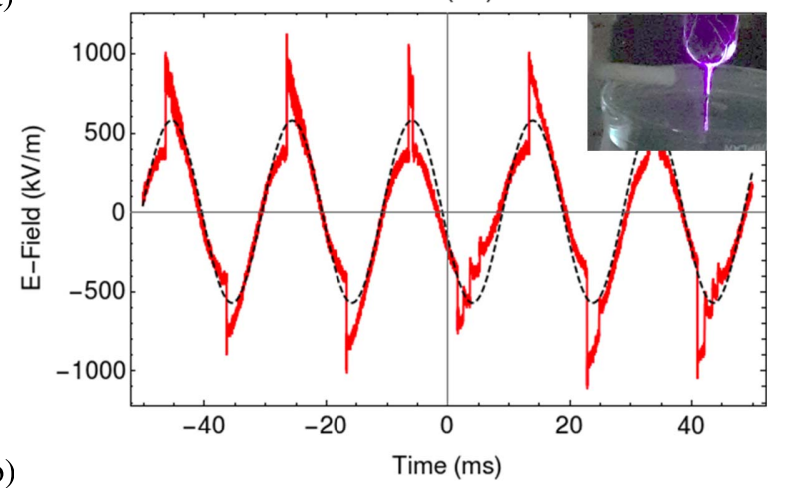

Fig. 4. Temporal evolution of the radial $E$-field. (a) Result for a feeding voltage of $16 \mathrm{kVrms}$. (b) Result for a feeding voltage of $18 \mathrm{kVrms}$. The $E$-field magnitude is shown with a continuous red line curve. The sine fit curve is represented by a black dashed line.

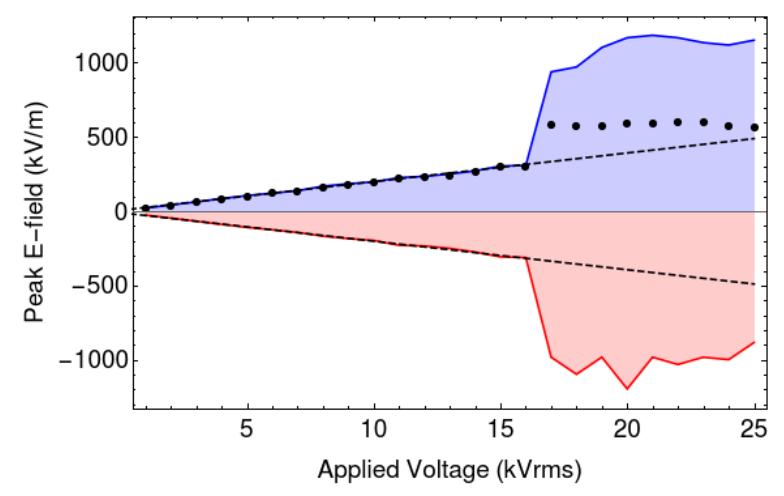

Fig. 5. Peak E-field induced as a function of the applied voltage. Blue: positive peak values. Red: negative peak values. Dashed lines linked to the fitting curves of the linear zone before the threshold. Black dots represent the amplitude of the radial electric field at $50 \mathrm{~Hz}$ as a function of the applied voltage.

Fig. 5 shows the peak values of measured $E$-field versus the applied voltage with 1-kVrms increment, from 1 to $25 \mathrm{kVrms}$. Up to $16 \mathrm{kVrms}$, the measured component behaves linearly with the applied voltage as expected in the absence of DBD.

Above $16 \mathrm{kVrms}$, corresponding to a field greater than $1 \mathrm{MV} / \mathrm{m}$ in between the electrodes, the peak value dramatically increases, being the signature of discharge-induced radial component (see the blue curve of Fig. 5 and inset on the top left of Fig. 1). Black dots in Fig. 5 represent the magnitude of the radial field at $50 \mathrm{~Hz}$ at different voltage values. These latter ones exhibit also a nonlinearity. This graph is divided into two

(c) 2021 IEEE. Personal use of this material is permitted. Permission from IEEE must be obtained for all other uses, in any current or future media, including reprinting/republishing this material for advertising or promotional purposes, creating new collective works, for resale or redistribution to servers or lists, or reuse of any copyrighted component of this work in other works. 


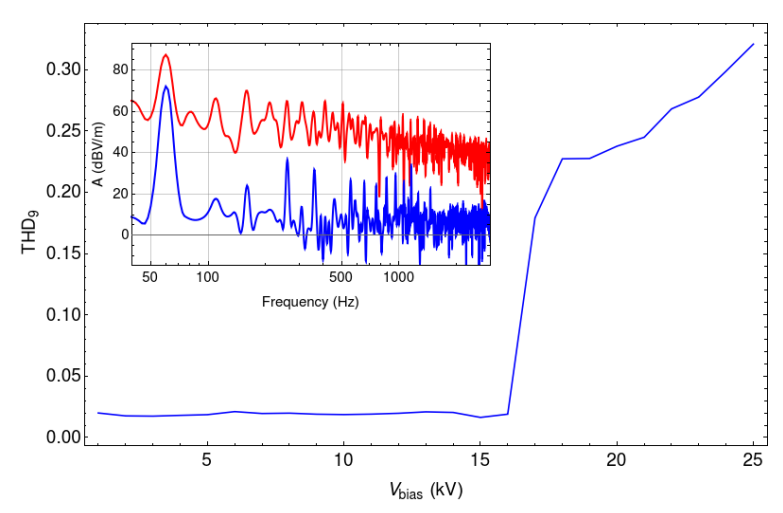

Fig. 6. THD versus the applied voltage. Inset: spectral distribution of temporal waveforms for applied voltage of $5 \mathrm{kV}$ (blue curve) and $20 \mathrm{kV}$ (red curve).

parts: from 0 to $16 \mathrm{kVrms}$, where the field increases linearly and gradually to reach a value of $300 \mathrm{kV} / \mathrm{m}$, and suddenly above $16 \mathrm{kVrms}$, there is a jump to reach a value twice larger than before $(600 \mathrm{kV} / \mathrm{m})$ and remains approximately constant beyond this voltage value. After the threshold, the amplitude of the 50-Hz signal remains almost constant (see black dots of Fig. 5). This means that the appearance of the filamentary discharges, for a given threshold value, limits the increase of nominal field with the increasing voltage. As the $50-\mathrm{Hz}$ field strength, the response is quite flat between 17 and $25 \mathrm{kV}$, meaning that the DBDs appear with a quite good reproducibility, at the same breakdown voltage or breakdown field.

Considering the geometry of the DBD reactor and regarding previous results, a voltage threshold of $17 \mathrm{kVrms}$ separates what we call in the following sections, the "low voltage" (no discharge) and the "high voltage" (with discharge) regimes, as shown in Fig. 5.

Below the threshold, the measured $E$-field is a $50-\mathrm{Hz}$ sinewave signal proportional to the applied voltage, while above the voltage threshold, spikes appear in the sine wave leading to harmonic distortion. The harmonic analysis, using numerical fast Fourier transform, is also proposed as a quantitative signature of the development of the ionization wave across the gap of the DBD reactor.

This discharge-induced behavior is clearly shown in Fig. 6, which exhibits the harmonic distortion as a function of the applied voltage. The total harmonic distortion (THD) is here defined up to the ninth harmonics of the signal and calculated through the following equation:

$$
\mathrm{THD}_{9}=\frac{\sqrt{\sum_{i=2}^{9} V_{i}^{2}}}{V_{50 \mathrm{~Hz}}}
$$

where $V_{i}$ corresponds to the magnitude of the $i$ th harmonic of the distorted $50-\mathrm{Hz}$ sine wave. This measurement concerns the analysis of transient signals and discharges initiated by the needle. This analysis can be carried out due to the flat response of the EO system over the frequency bandwidth of interest.

The signal spectrum is then strongly modified with a significant enhancement of spectral components exceeding the fundamental frequency of $50 \mathrm{~Hz}$, induced by the discharge.

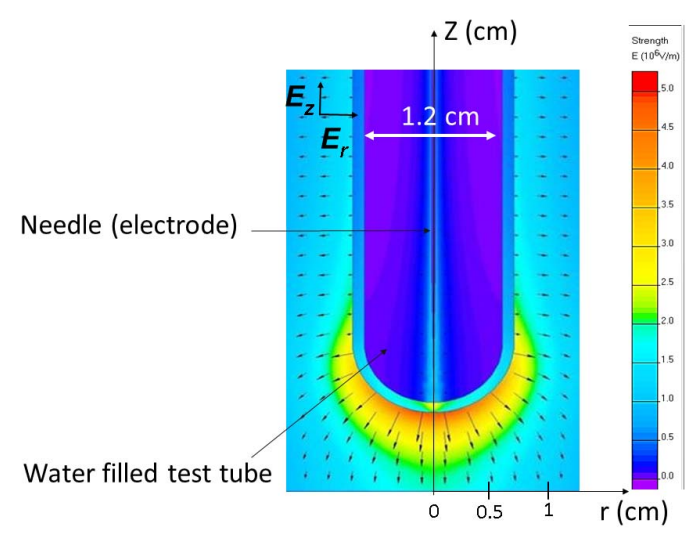

Fig. 7. Spatial distribution of the electric field with the structure under test at $17 \mathrm{kVrms}$.

Indeed, the calculation shows a THD of about $2 \%$ below the threshold, which corresponds to the THD of the high voltage source. This THD exceeds more than $22 \%$ over the threshold and reaches even $32 \%$ for an applied voltage of $25 \mathrm{kVrms}$.

The above-described behavior can be predicted by an electrostatic simulation as long as we stay in the low-voltage regime (in the absence of discharge). The DBD reactor, described in Section II, has been simulated with the finite element method (using QuickField software) and meshed with more than 300000 nodes. The result is presented in Fig. 7.

The dielectric permittivity and geometry of all constituting elements are considered in this virtual reactor (metallic needle and ground plate, glass tube, Petri dish, air, and water). Under the conditions corresponding to the experimental parameters of Fig. 1, the model predicts an appearance of the plasma when the electric field value is greater than the breakdown value in the air (3 MV/m [50]). The measured threshold of DBD appearance at $16.5 \mathrm{kVrms}$ is in agreement with the numerical expectations shown in Fig. 7, with an expected threshold of $17 \mathrm{kVrms}$.

\section{B. 1-D Mapping of the Radial Field}

Temporal evolution of the radial electric field around the discharge is measured for different positions of the probe. Measurements have been performed every $5 \mathrm{~mm}$ from $0 \mathrm{~cm}$ (within the discharge) to $10 \mathrm{~cm}$ away (cf Fig. 7) along the $r$-axis, where the probe is placed between the two electrodes. The applied voltage has been set to $23 \mathrm{kVrms}$ in order to generate discharge in between the electrodes of the DBD reactor. The raw measurement is presented in Fig. 8, with a representation of the temporal traces for each position of the probe.

Two different zones can be clearly observed. From 0 to $2 \mathrm{~cm}$, this is the area associated with the intense electric field, with a typical signature of the ionization waves (see red and purple zone in Fig. 8). After $2 \mathrm{~cm}$, for remote distance from the discharge, only contributions of the Laplacian field (radial field induced by the needle) are seen.

A further analysis is proposed in Fig. 9. It shows the spatial distribution of the radial component and its maximum. 


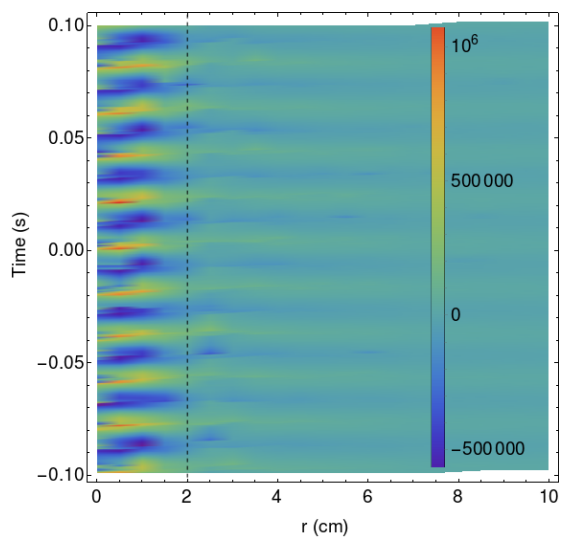

Fig. 8. Experimental 1-D mapping of the temporal waveform associated with the radial component of the $E$-field at $23 \mathrm{kVrms}$.

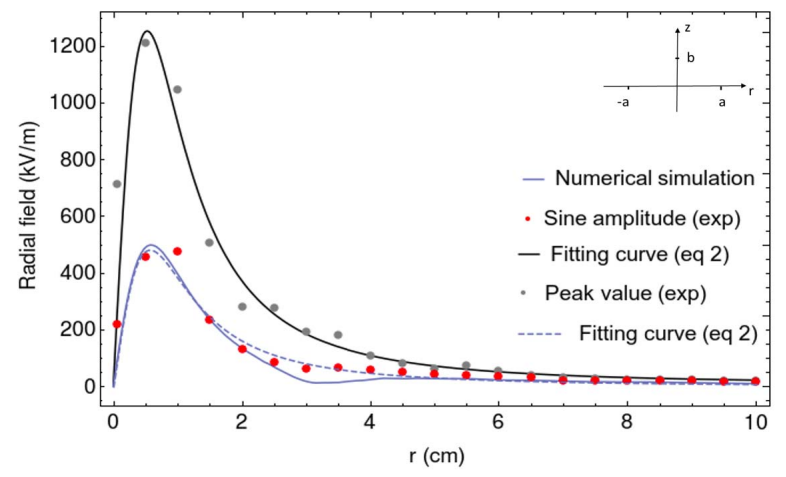

Fig. 9. Spatial distribution of the radial component of the field. Data obtained at $23 \mathrm{kVrms}$ and each $5 \mathrm{~mm}$.

Experimental results are represented and compared with analytical calculations and numerical simulations.

Note that the theoretical equation is given by calculation of the spatial derivative of the potential between a point-plane configuration (in two dimensions) based on the electrostatic equations

$$
\begin{aligned}
E(r, z)= & \frac{2 r}{\left(r^{2}+(z-b)^{2}\right)^{3 / 2}} \\
& +\frac{\frac{r-a}{\sqrt{(r-a)^{2}+z^{2}}}+1}{\sqrt{(r-a)^{2}+z^{2}}+r-a} \\
& -\frac{\frac{r+a}{\sqrt{(r+a)^{2}+z^{2}}}+1}{\sqrt{(r+a)^{2}+z^{2}}+r+a}
\end{aligned}
$$

where $a$ is the radius of the plane, as indicated in the inset of Fig. 9, and $b$ is about $8 \mathrm{~mm}$, and it corresponds to the pointplane distance, as shown in Fig. 1. When $a$ tends to infinity, it gives

$$
E(r, z)=\frac{2 r}{\left(r^{2}+(z-b)^{2}\right)^{3 / 2}}
$$

In Fig. 9, zero corresponds to the position of the DBD, which shows no radial field (vertical $E$-field), and then, we move the probe, each $0.5 \mathrm{~cm}$, from the DBD until the electric field reaches a value of $500 \mathrm{kV} / \mathrm{m}$ at $1 \mathrm{~cm}$ away

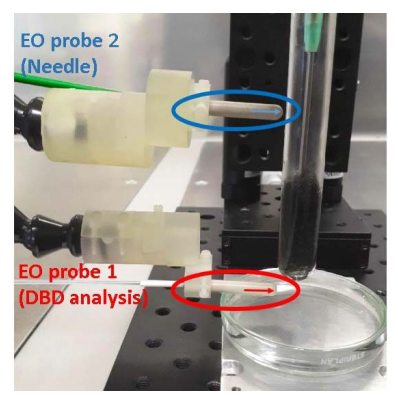

Fig. 10. Photograph showing the two EO probes with their sensibility axis (colored arrows indicated on the probe packaging).

from the discharge. Also, by moving further and further away from the discharge, the field gradually decreases and vanishes as expected. A good agreement between experiment, theory, and simulation, for field distribution and absolute values, is observed.

\section{Additional Experiment Campaigns Involving Two EO Probes}

1) Contribution of Needle and DBDs to the Field: The goal of the below-described measurement campaign is to identify the $E$-field from the filamentary discharge with the Laplacian field from the needle electrode, on the one hand, within the electrodes gap, on the other hand. This would lead to measure the $E$-field contribution of discharge generated by the DBD reactor configuration.

For that purpose, two separated longitudinal EO probes (i.e., sensitive to the $E$-field component aligned with the probe axis) were positioned such that they both give access independently to the radial component of the radiated $E$-field (see Fig. 10). In such configuration (see Fig. 10), probe 2 provides the applied ac voltage, while probe 1 leads simultaneously to the knowledge of the $E$-field in between the electrodes. Such experimental configuration has led to the signals presented in Fig. 11(a) (in the absence of discharges) and Fig. 11(b) (with discharges).

According to Fig. 11, the signals delivered simultaneously by the two EO probes, both calibrated to get the absolute value of the $E$-field, depend on the magnitude of ac voltages applied to the DBD reactor. For relatively low voltage [Fig. 11(a)], the signal measured within the electrodes gap (blue curve) has a lower amplitude than the one radiated from the needle (red curve). Indeed, considering the $E$-field lines and strength shown by the simulation (see Fig. 7), it is obvious that the radial components measured by both probes are smaller in between the electrodes gap than laterally close to the needle. This is no longer true when high voltage is applied to the DBD reactor as shown in Fig. 11(b), meaning that the discharge filament propagating across the gap is increasing virtually the needle length. The outcome of the electrostatic simulation of a filamentary conductive channel is in agreement with the results presented in Section III-B.

In addition, to the discharge-induced disturbances above described, one notices that the radial components simultaneously measured by the two EO probes are in phase in high- 


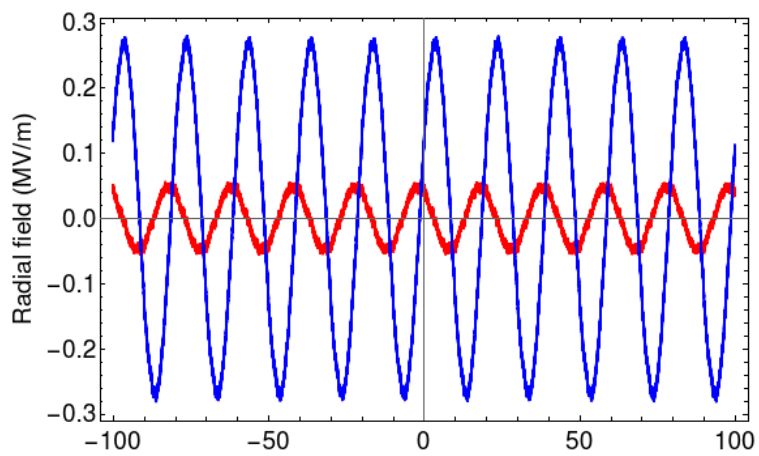

a)

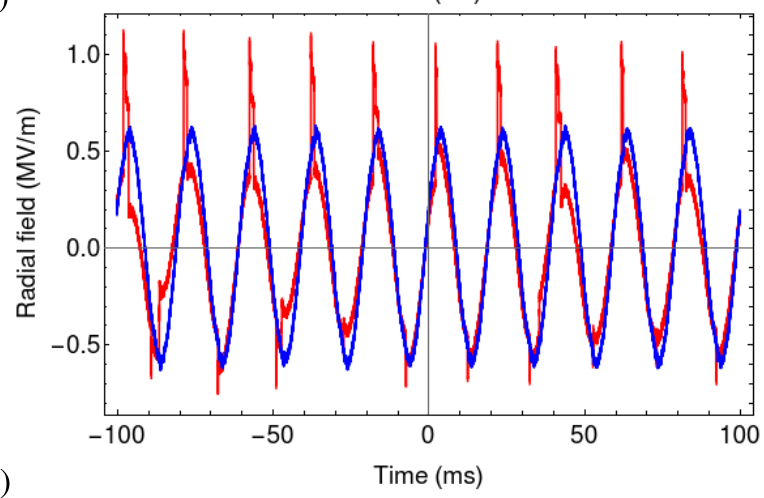

Fig. 11. Temporal evolution of the radial field. Red and blue curves correspond to probe 1 (closed to DBD location) and probe 2 (higher position, closed to the top of the needle), respectively. (a) Result for a feeding voltage of $11 \mathrm{kVrms}$. (b) Result for a feeding voltage of $22 \mathrm{kVrms}$.

voltage regime, while they are in quadrature at a lower voltage (below voltage threshold). This is explained by considering the electrical equivalent circuit of the discharge reactor. Indeed, without discharge, the reactor is mainly a capacitor presenting a pure imaginary impedance. In turn, this capacitive reactor at low voltage introduces a $(\pi / 2)$ phase shift in between the applied voltage measured by the EO probe 2 (blue curve) and the $E$-field measured by probe 1 (red curve). In the highvoltage regime, it is different (see Fig. 11). When the applied voltage is high enough to initiate a breakdown, a conducting channel is set from one electrode to the other. This channel is electrically equivalent to a conducting wire whose resistance depends on the characteristics of the DBD reactor (type of barriers/target, dielectric properties, and shape of the interelectrodes media...). The reactor turns mainly into a resistive equivalent circuit presenting a real impedance: almost no phase shift is seen between red and blue curves of Fig. 11(b).

Consequently, as the $E$-field radiated by a wire-equivalent discharge aligned from the needle tip to the ground is mainly radial, the peaks observed in Fig. 11(b) originate from the discharge. This supports the hypothesis made in Section III-C1.

2) Polarimetric Analysis of the Field: Due to the excellent rejection of orthogonal $E$-field components to the one probe by the EO sensor, a real-time polarimetric analysis can therefore be performed. The synchronized acquisition of the radial and longitudinal components $\left(E_{r}\right.$ and $E_{z}$ like shown in Fig. 12) gives access to the $E$-field vector and its temporal evolution leads in turn to its polarization state. The inset of Fig. 12 gives photography of the two probes.

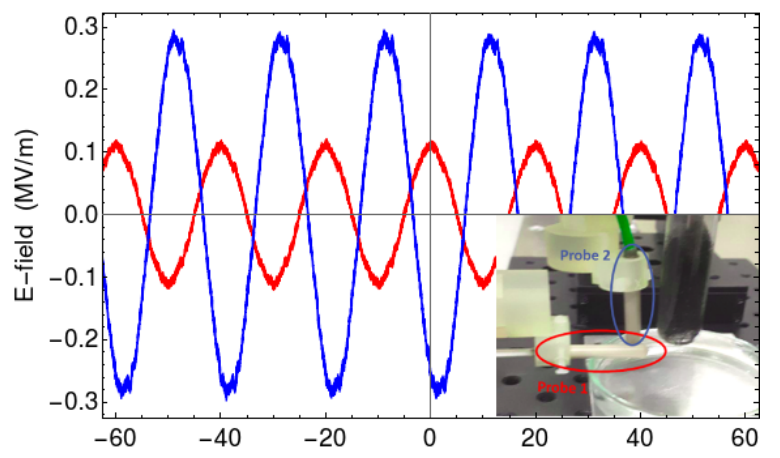

a)

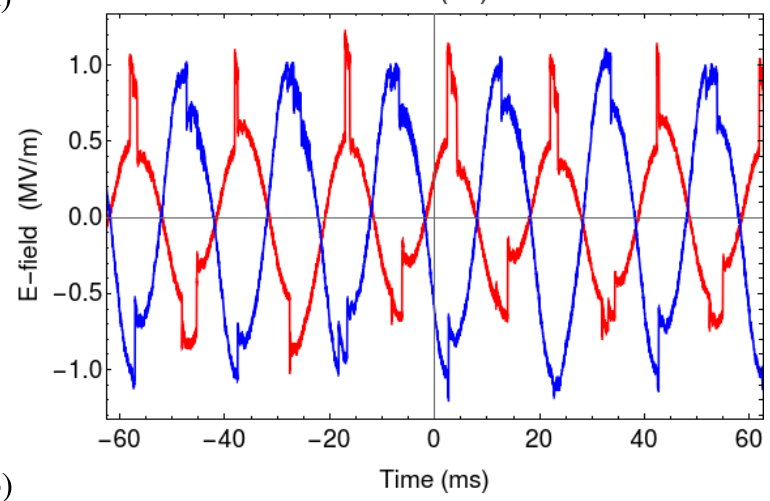

Fig. 12. Temporal evolution of the two components of the field. Red and blue curves correspond to $E_{r}$ and $E_{z}$, respectively. (a) Result for a feeding voltage of $10 \mathrm{kVrms}$. (b) Result for a feeding voltage of $23 \mathrm{kVrms}$.

Fig. 12 shows some examples of temporal measurements required to obtain the polarization state of the field in between the needle and the ground plane. Two applied voltages, below and above the threshold, are investigated. Under $16 \mathrm{kVrms}$, a phase shift of $\sim(\pi / 2)$ is observed between $E_{r}$ and $E_{z}$. Indeed, the measurement of $E_{z}$ with probe 2 leads to the direct image of the potential of the needle. Probe 1, with its sensitivity axis, along $E_{r}$ is located just below probe 2 (in the capacitance area, just below the bottom of the electrode under voltage). This position is clearly chosen to improve the detection of DBDs, which dramatically influences $E_{r}$. When the applied voltage (here $23 \mathrm{kVrms}$ ) exceeds the threshold, one can notice that the appearance of DBDs induces a phase shift between $E_{r}$ and $E_{z}$ (output signal and probe direction are correlated). Once again, concerning the $E_{r}$ component, a voltage multiplied by a factor of two leads to field values multiplied by 5 , due to the contribution of DBDs induced charges. Another representation is proposed for the synchronized two-component measurement. This is shown in Fig. 13. Here, parametric plots of $E_{z}$ as a function of $E_{r}$ allow getting the polarimetric behavior of the field for different values of the applied voltage. Fig. 13(a) corresponds to configurations with too low voltages for DBD generation. Due to the phase shift between the two eigencomponents of the field, the polarimetric figures describe ellipses. This elliptical shape of the field pattern is mainly vertical [phase shift of $\sim(\pi / 2)$ ] due to the location of the measurement (in between the two electrodes). Three different voltages are here considered and the associated obtained ellipses are proportional to each 
a)

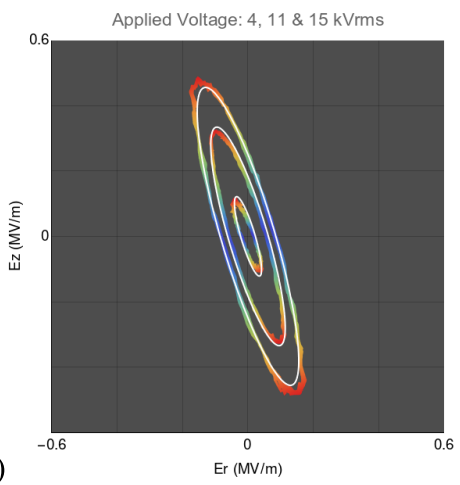

b)

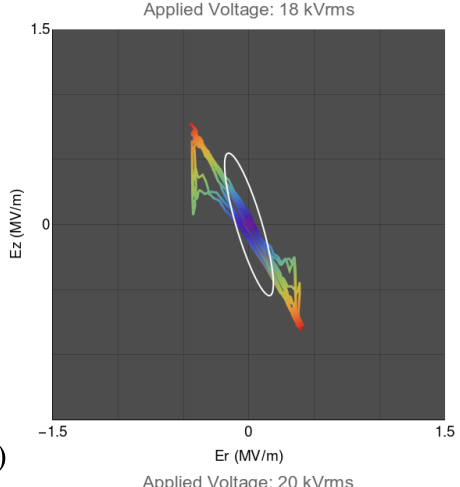

c)

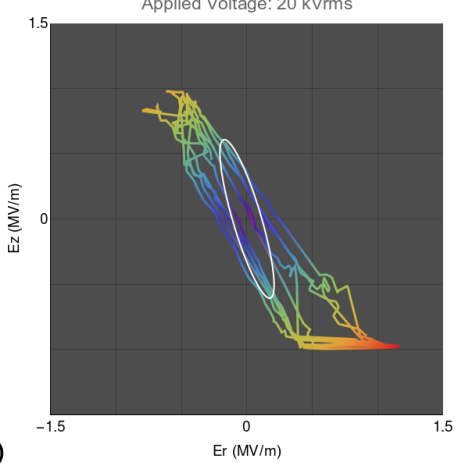

Applied Voltage: $24 \mathrm{kVrms}$

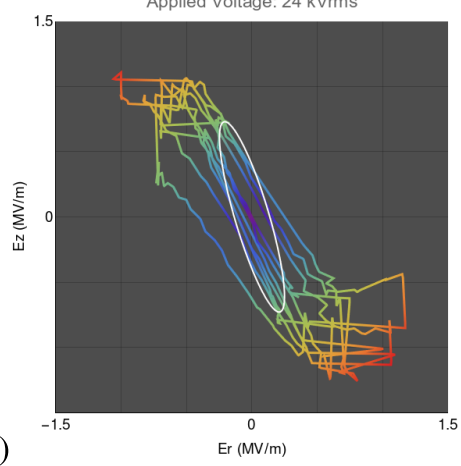

Fig. 13. Temporal trace of the $E$-field vector calculated from the measurements of $E_{r}$ and $E_{z}$ for an applied voltage of (a) 4, 11, and $15 \mathrm{kVrms}$ and (b) 18 , (c) 20, and (d) $24 \mathrm{kVrms}$.

other. Their ellipticity remains the same and their sizes are linearly linked to the feeding voltage. After the threshold, we can notice a dramatic difference between the signature with and without DBD. Once the breakdown has occurred, there is no ellipsoidal behavior, and the shape is defined by several almost straight lines [see the blue and green zones of Fig. 13(b)-(d)]. This illustrates a proportional link between
$E_{r}$ and $E_{z}$, with linear coefficients depending slightly on the considered period of the temporal waveform. The slope $\left|E_{z} / E_{r}\right|$ ranges from 0.9 to 1.6. Nevertheless, a comparison between the three polarization states above $17 \mathrm{kVrms}$ clearly shows that the higher the voltage is, the wider the space between the straight lines. This can be explained by the fact that for a high value of applied voltage, the instantaneous required field strength for the generation of ionization wave differs dramatically from one period to another. Moreover, the higher the voltage is, the higher the dispersion for the discharge location in the $\left\{E_{r}, E_{z}\right\}$ space [see the orange and red zones of Fig. 13(b)-(d)]. Finally, white ellipses (linearly scaled from measurement without DBD) have been superimposed to the actual polarimetric measurement to easily identify the qualitative and quantitative influences of the DBDs themselves on the field pattern. For lower values of the field strength [Fig. 13(a)], $E_{r}$ increases linearly together with $E_{z}$. The slope $\left|E_{r} / E_{z}\right|$ ranges from 0.62 to 1.16 . The different color from blue to red presents the low to the high value of the $E$-field. One notices a significant difference between the signature with and without discharge.

\section{CONCLUSiON}

In this work, we have used EO probes to perform a comprehensive analysis of the $E$-field induced by a filamentary discharge in a DBD configuration. The measured threshold for discharge breakdown generation is determined and it is in good agreement with the electrostatic simulation. A quantitative assessment of nonlinear behavior between the field and the applied voltage is demonstrated. Moreover, the real-time evolution of the two eigenfield vector components induced by the high voltage source and the ionization waves propagating within the electrodes gap of the DBD reactors has been carried out. A specific polarimetric signature of filamentary discharge is here identified.

\section{REFERENCES}

[1] A. Fridman, A. Chirokov, and A. Gutsol, "Non-thermal atmospheric pressure discharges," J. Phys. D, Appl. Phys., vol. 38, no. 2, pp. R1-R24, Jan. 2005.

[2] F. Judée, S. Simon, C. Bailly, and T. Dufour, "Plasma-activation of tap water using DBD for agronomy applications: Identification and quantification of long lifetime chemical species and production/consumption mechanisms," Water Res., vol. 133, pp. 47-59, Apr. 2018.

[3] I. Adamovich et al., "The 2017 plasma roadmap: Low temperature plasma science and technology," J. Phys. D, Appl. Phys., vol. 50, no. 32, Aug. 2017, Art. no. 323001.

[4] M. Xi, Y.-L. Li, S.-Y. Shang, D.-H. Li, Y.-X. Yin, and X.-Y. Dai, "Surface modification of aramid fiber by air DBD plasma at atmospheric pressure with continuous on-line processing," Surf. Coatings Technol., vol. 202, no. 24, pp. 6029-6033, Aug. 2008.

[5] L. Wei, T. Xiaodong, L. Dongping, L. Yanhong, F. Zhiqing, and C. Baoxiang, "Growth of fluorocarbon films by low-pressure dielectric barrier discharge," Plasma Sci. Technol., vol. 10, no. 1, pp. 74-77, Feb. 2008.

[6] F. Massines, N. Gherardi, and F. Sommer, "Silane-based coatings on polypropylene, deposited by atmospheric pressure glow discharge plasmas," Plasmas Polym., vol. 5, nos. 3-4, pp. 151-172, 2000.

[7] G.-M. Xu, Y. Ma, and G.-J. Zhang, "DBD plasma jet in atmospheric pressure argon,” IEEE Trans. Plasma Sci., vol. 36, no. 4, pp. 1352-1353, Aug. 2008.

(C) 2021 IEEE. Personal use of this material is permitted. Permission from IEEE must be obtained for all other uses, in any current or future media, including reprinting/republishing this material for advertising or promotional purposes, creating new collective works, for resale or redistribution to servers or lists, or reuse of any copyrighted component of this work in other works. 
[8] P. Rajasekaran, P. Mertmann, N. Bibinov, D. Wandke, W. Viöl, and P. Awakowicz, "Filamentary and homogeneous modes of dielectric barrier discharge (DBD) in air: Investigation through plasma characterization and simulation of surface irradiation," Plasma Processes Polym., vol. 7, no. 8, pp. 665-675, Aug. 2010.

[9] Z. Navrátil et al., "Comparative study of diffuse barrier discharges in neon and helium," Plasma Sour. Sci. Technol., vol. 15, no. 1, pp. 8-17, Feb. 2006.

[10] U. Kogelschatz, "Filamentary, patterned, and diffuse barrier discharges," IEEE Trans. Plasma Sci., vol. 30, no. 4, pp. 1400-1408, Aug. 2002.

[11] T. Shao et al., "Electrical characterization of dielectric barrier discharge driven by repetitive nanosecond pulses in atmospheric air," J. Electrostatics, vol. 67, nos. 2-3, pp. 215-221, May 2009.

[12] Y.-M. Sung and T. Sakoda, "Optimum conditions for ozone formation in a micro dielectric barrier discharge," Surf. Coatings Technol., vol. 197, nos. 2-3, pp. 148-153, Jul. 2005.

[13] J. M. Williamson, D. D. Trump, P. Bletzinger, and B. N. Ganguly, "Comparison of high-voltage AC and pulsed operation of a surface dielectric barrier discharge," J. Phys. D, Appl. Phys., vol. 39, no. 20, pp. 4400-4406, Oct. 2006.

[14] A. Bogaerts et al., "The 2020 plasma catalysis roadmap," J. Phys. D, Appl. Phys., vol. 53, no. 44, Oct. 2020, Art. no. 443001.

[15] A. Khacef, J. M. Cormier, and J. M. Pouvesle, " $\mathrm{NO}_{x}$ remediation in oxygen-rich exhaust gas using atmospheric pressure nonthermal plasma generated by a pulsed nanosecond dielectric barrier discharge," J. Phys. D, Appl. Phys., vol. 35, no. 13, pp. 1491-1498, Jul. 2002.

[16] M. Magureanu, N. B. Mandache, E. Gaigneaux, C. Paun, and V. I. Parvulescu, "Toluene oxidation in a plasma-catalytic system," J. Appl. Phys., vol. 99, no. 12, Jun. 2006, Art. no. 123301.

[17] R. P. Mildren, R. J. Carman, and I. S. Falconer, "Visible and VUV emission from a Xenon dielectric barrier discharge using pulsed and sinusoidal voltage excitation waveforms," IEEE Trans. Plasma Sci., vol. 30, no. 1, pp. 192-193, Feb. 2002.

[18] R. J. Carman, R. P. Mildren, B. K. Ward, and D. M. Kane, "Highpressure ( $>1$ bar) dielectric barrier discharge lamps generating short pulses of high-peak power vacuum ultraviolet radiation," J. Phys. D, Appl. Phys., vol. 37, no. 17, pp. 2399-2407, Sep. 2004.

[19] S. Beleznai, G. Mihajlik, I. Maros, L. Balázs, and P. Richter, "Improving the efficiency of a fluorescent Xe dielectric barrier light source using short pulse excitation," J. Phys. D, Appl. Phys., vol. 41, no. 11, Jun. 2008, Art. no. 115202 .

[20] S. Ishikawa, K. Yukimura, K. Matsunaga, and T. Maruyama, "The surface modification of poly (tetrafluoroethylene) film using dielectric barrier discharge of intermittent pulse voltage," Surf. Coatings Technol., vol. 130 , no. 1 , pp. 52-56, Aug. 2000.

[21] A. N. Bhoj and M. J. Kushner, "Repetitively pulsed atmospheric pressure discharge treatment of rough polymer surfaces: I. Humid air discharges," Plasma Sour. Sci. Technol., vol. 17, no. 3, Aug. 2008, Art. no. 035024.

[22] J. L. Walsh, J. J. Shi, and M. G. Kong, "Contrasting characteristics of pulsed and sinusoidal cold atmospheric plasma jets," Appl. Phys. Lett., vol. 88, no. 17, Apr. 2006, Art. no. 171501.

[23] P. Magnier, B. Dong, D. Hong, and J. Hureau, "Action of a pulsed DBD actuator on a slow jet," J. Electrostatics, vol. 66, nos. 7-8, pp. 369-374, Jul. 2008.

[24] B. L. Sands, B. N. Ganguly, and K. Tachibana, "A streamer-like atmospheric pressure plasma jet," Appl. Phys. Lett., vol. 92, no. 15, Apr. 2008, Art. no. 151503

[25] X. Lu, Z. Jiang, Q. Xiong, Z. Tang, and Y. Pan, "A single electrode room-temperature plasma jet device for biomedical applications," Appl. Phys. Lett., vol. 92, no. 15, Apr. 2008, Art. no. 151504.

[26] J. H. Choi et al., "Analysis of sterilization effect by pulsed dielectric barrier discharge," J. Electrostat., vol. 64, no. 1, pp. 17-22, 2006.

[27] B. Gellert and U. Kogelschatz, "Generation of excimer emission in dielectric barrier discharges," Appl. Phys. B, Photophysics Laser Chem., vol. 52, no. 1, pp. 14-21, Jan. 1991.

[28] U. Kogelschatz, "Advanced ozone generation," in Process Technologies for Water Treatment. Boston, MA, USA: Springer, 1988, pp. 87-118.

[29] P. J. Bruggeman, M. J. Kushner, B. R. Locke, J. G. E. Gardeniers, W. G. Graham, and D. B. Graves, "Plasma-liquid interactions: A review and roadmap," Plasma Sources Sci. Technol., vol. 25, no. 5, 2016, Art. no. 053002
[30] P. Vanraes et al., "Removal of several pesticides in a falling water film DBD reactor with activated carbon textile: Energy efficiency," Water Res., vol. 116, pp. 1-12, Jun. 2017.

[31] E. Marotta, E. Ceriani, M. Schiorlin, C. Ceretta, and C. Paradisi, "Comparison of the rates of phenol advanced oxidation in deionized and tap water within a dielectric barrier discharge reactor," Water Res., vol. 46, no. 19, pp. 6239-6246, Dec. 2012.

[32] M. Magureanu, N. B. Mandache, and V. I. Parvulescu, "Degradation of pharmaceutical compounds in water by non-thermal plasma treatment," Water Res., vol. 81, pp. 124-136, Sep. 2015.

[33] N. Korichi, O. Aubry, H. Rabat, B. Cagnon, and D. Hong, "Paracetamol degradation by catalyst enhanced non-thermal plasma process for a drastic increase in the mineralization rate," Catalysts, vol. 10, no. 9, p. 959 , Aug. 2020.

[34] W. D. Prather, C. E. Baum, R. J. Torres, F. Sabath, and D. Nitsch, "Survey of worldwide high-power wideband capabilities," IEEE Trans. Electromagn. Compat., vol. 46, no. 3, pp. 335-344, Aug. 2004.

[35] S. Kharkovsky and R. Zoughi, "Microwave and millimeter wave nondestructive testing and evaluation-Overview and recent advances," IEEE Instrum. Meas. Mag., vol. 10, no. 2, pp. 26-38, Apr. 2007.

[36] H. W. Ott, Electromagnetic Compatibility Engineering. Hoboken, NJ, USA: Wiley, 2009.

[37] T. Iyama, K. Kiminami, and T. Onishi, "Applicability of three-axis electro-optic (EO) probe for specific absorption rate (SAR) measurement," IEICE Trans. Commun., vol. 92, no. 4, pp. 1414-1417, 2009.

[38] G. Gaborit et al., "A nonperturbative electrooptic sensor for in situ electric discharge characterization," IEEE Trans. Plasma Sci., vol. 41, no. 10, pp. 2851-2857, Oct. 2013.

[39] G. Gaborit et al., "Single shot and vectorial characterization of intense electric field in various environments with pigtailed electrooptic probe," IEEE Trans. Plasma Sci., vol. 42, no. 5, pp. 1265-1273, May 2014.

[40] Y. Gaeremynck, G. Gaborit, L. Duvillaret, M. Ruaro, and F. Lecoche, "Two electric-field components measurement using a 2-port pigtailed electro-optic sensor," Appl. Phys. Lett., vol. 99, no. 14, Oct. 2011, Art. no. 141102.

[41] G. Gaborit et al., "Pockels' effect-based probe for UWB and HPEM measurements," in Ultra-Wideband, Short-Pulse Electromagnetics. New York, NY, USA: Springer, 2014, pp. 411-421.

[42] A. Schmidt-Bleker, J. Winter, S. Iseni, M. Dünnbier, K.-D. Weltmann, and S. Reuter, "Reactive species output of a plasma jet with a shielding gas device-Combination of FTIR absorption spectroscopy and gas phase modelling," J. Phys. D, Appl. Phys., vol. 47, no. 14, Apr. 2014, Art. no. 145201.

[43] V. V. Kovacevic, B. P. Dojcinovic, M. Jovic, G. M. Roglic, B. M. Obradovic, and M. M. Kuraica, "Measurement of reactive species generated by dielectric barrier discharge in direct contact with water in different atmospheres," J. Phys. D, Appl. Phys., vol. 50, no. 15, Apr. 2017, Art. no. 155205.

[44] V. V. Kovacevic, G. B. Sretenovic, E. Slikboer, O. Guaitella, A. Sobota, and M. M. Kuraica, "The effect of liquid target on a nonthermal plasma jet-Imaging, electric fields, visualization of gas flow and optical emission spectroscopy," J. Phys. D, Appl. Phys., vol. 51, no. 6, Feb. 2018, Art. no. 065202.

[45] L. Duvillaret, S. Rialland, and J. Coutaz, "Electro-optic sensors for electric field measurements. II. Choice of the crystals and complete optimization of their orientation," J. Opt. Soc. Amer. B, Opt. Phys., vol. 19, no. 11, pp. 2704-2715, 2002.

[46] L. Duvillaret, S. Rialland, and J. L. Coutaz, "Electro-optic sensors for electric field measurements. I. Theoretical comparison among different modulation techniques," J. Opt. Soc. Amer. B, Opt. Phys., vol. 19, no. 11, pp. 2692-2703, 2002.

[47] G. B. Sretenovic̀ et al., "Electric field measurement in the dielectric tube of helium atmospheric pressure plasma jet," J. Appl. Phys., vol. 121, no. 12, Mar. 2017, Art. no. 123304.

[48] T. L. Chng, A. Brisset, P. Jeanney, S. M. Starikovskaia, I. V. Adamovich, and P. Tardiveau, "Electric field evolution in a diffuse ionization wave nanosecond pulse discharge in atmospheric pressure air," Plasma Sour. Sci. Technol., vol. 28, no. 9, Sep. 2019, Art. no. 09LT02.

[49] M. Bernier, G. Gaborit, L. Duvillaret, A. Paupert, and J.-L. Lasserre, "Electric field and temperature measurement using ultra wide bandwidth pigtailed electro-optic probes," Appl. Opt., vol. 47, no. 13, p. 2470, 2008.

[50] K. H. Becker, U. Kogelschatz, K. H. Schoenbach, and R. J. Barker, Non-Equilibrium Air Plasmas at Atmospheric Pressure. Boca Raton, FL, USA: CRC Press, 2004. 


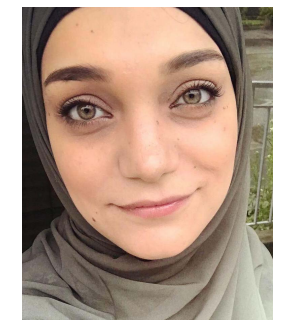

Farah Aljammal was born in Lebanon in 1994. She received the M.Sc. degree in condensed matter physics from Lebanese University, Beirut, Lebanon, in 2017. She is currently pursuing the Ph.D. degree in optics and radio frequency with the IMEP-LAHC laboratory, Grenoble Alpes University, Grenoble, France. Her thesis title is "Nonlinear Optics and Plasmas: Electric Field Characterization and $\mathrm{THz}$ Generation."

Her research work mainly focuses on the vectorial and noninvasive characterization of the electric field associated with cold atmospheric plasmas (dielectric barrier discharge, plasma jet, and so on). The other part of her doctoral work is devoted to the nonlinear interaction between a cold plasma and a pulsed optical wave for the parametric generation of terahertz waves.

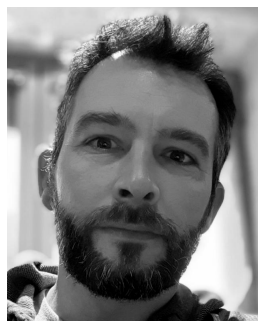

Gwenaël Gaborit was born in France in 1978. $\mathrm{He}$ received the $\mathrm{Ph} . \mathrm{D}$. degree in physics from the University of Savoie, Chambéry, France, in 2005.

Since 2006, he has been an Associate Professor with the IMEP-LAHC laboratory, University SavoieMont-Blanc, Le Bourget-du-Lac, France. He is also a Co-Founder and a Chief Science Officer of Kapteos S.A.S, Sainte-Hélène-du-Lac, France. He passed the French Accreditation to Supervise Research (HDR) in 2014. He has coauthored over 100 journal articles and international conference proceedings. He holds four patents. His main research interest focuses on electrooptic sensors dedicated to noninvasive electric field characterization and terahertz polarimetric analysis. The wide range of applications associated to his research includes defense, healthcare, energy, telecom, and aerospace.

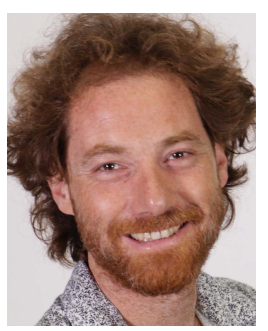

Maxime Bernier was born in France in 1980. $\mathrm{He}$ received the M.Sc. degree in matter and radiation from Burgundy University, Besançon, France, in 2003, the M.Sc. degree in microwave and optical telecommunications from the University of Limoges, Limoges, France, in 2004, and the Ph.D. degree in optic and radiofrequencies from the Institut National Polytechnique of Grenoble, Grenoble, France, in 2008, for a work dedicated to electrooptic detection of $E$-field.

He spent two years as a Post-Doctoral Fellow with the Laboratory of Femtosecond Spectroscopy, University of Sherbrooke, Sherbrooke, Canada. Since September 2011, he has been an Assistant Professor at the IMEP-LAHC laboratory, University of Savie Mont Blanc, Le Bourgetdu-Lac, France, where his activities are related to terahertz signal generation and detection, and accurate materials and devices' characterization.

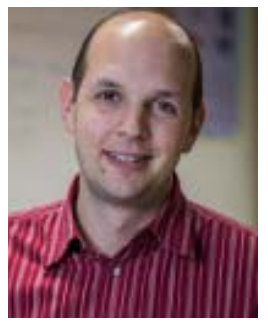

Sylvain Iséni was born in France in 1988. $\mathrm{He}$ received the M.Sc. degree in physics from the University of Orléans, Orléans, France, in 2011, and the M.Eng. degree from the École Polytechnique de l'Université d'Orléans, Orléans, with a focus on optics, laser, and plasma physics and processes. $\mathrm{He}$ was a Ph.D. candidate with the Center for Innovation Competence Plasmatis, Leibniz-Institut für Plasmaforschung und Technologie e.V. (INP Greifswald e.V.), Greifswald, Germany, and received the Ph.D. degree from Ernst-Moritz-Arndt-University,

Greifswald, in 2015.

His work focuses on laser diagnostics for low-temperature plasmas for biomedical applications. He became a Research Fellow at the Research Group for the Energetics of Ionized Media (GREMI), Orléans, from 2015 to 2017. Since 2017, he has been a Permanent Researcher with the French National Center for Scientific Research (CNRS), GREMI. His research focuses on the nonequlibrium-nonthermal plasmas and plasma processes significant for water, environmental, and biological purposes. His research focuses on many fields mainly dealing with optical emission spectroscopy, laser spectroscopy, imaging techniques, as well as electrical diagnostics.

Dr. Iséni is a member of the International Low Temperature Plasma Community (ILTPC), the CNRS Réseau Plasmas Froids, as well as the CNRS GDR EMILI-Etude des MILieux Ionisés: Plasmas Froids créées par décharge et laser.

Laurence Galtier was born in France in 1989. She received the M.Eng. degree from the Institut d'Optique Graduate School, Palaiseau, France, in 2014, and the M.Sc. degree in optical vision and image analysis from Jean Monnet University, Saint-Étienne, France, in 2014.

For three years, she was an Optical Engineer working on electrooptic probe manufacturing dedicated to electric field measurement in harsh environments at Kapteos SAS, Sainte-Hélène-du-Lac, France. After working with Parrot to design optical cameras for UAVs, she is currently an Industrialization Engineer at Fibercryst, Lyon, France, which manufactures femtosecond lasers for industry with SCF amplifier technology, patented by this company.

Guillaume Revillod was born in France in 1978. He received the Ph.D. degree in physics from the University of Lyon, Lyon, France, in 2006.

He did a post-doctoral fellowship at Kyoto University, Kyoto, Japan, and then start to work as a Research and Development Engineer at HORIBA Headquarter. He came back in France to work in start-up developing breakthrough scientific measurement devices in a wide range of applications, such as energy, defense, chemicals, healthcare, and aerospace. He is currently the CTO of Kapteos, Sainte-Hélène-du-Lac, France, a company specialized in electromagnetic field measurement.

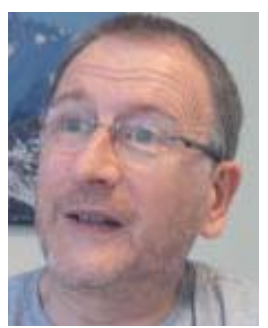

Lionel Duvillaret received the Ph.D. degree from Paris-Sud, Orsay, France, in 1994.

$\mathrm{He}$ has been an Associate Professor at Savoy University, Chambéry, France, up to 2005. Then, he became a Professor at the Grenoble Polytechnic Institute, Grenoble, France. He founded Kapteos SAS, Sainte-Hélène-du-Lac, France, in 2009, and he is currently its CEO. He has authored or coauthored dozens of patents and more than 190 articles and international conferences in the field of $E$-field measurements via electrooptic technology, terahertz time-domain spectroscopy, and electromagnetic bandgap devices. 